\title{
Association of comorbid mood disorders and chronic illness with disability and quality of life in Ontario, Canada
}

T Gadalla, PhD (1)

\begin{abstract}
Mood disorders are more prevalent in individuals with chronic physical illness compared to individuals with no such illness. These disorders amplify the disability associated with the physical condition and adversely affect its course, thus contributing to occupational impairment, disruption in interpersonal and family relationships, poor health and suicide. This study used data collected in the Canadian Community Health Survey, cycle 3.1 (2005) to examine factors associated with comorbid mood disorders and to assess their association with the quality of life of individuals living in Ontario. Results indicate that individuals with chronic fatigue syndrome, fibromyalgia, bowel disorder or stomach or intestinal ulcers had the highest rates of mood disorders. The odds of having a comorbid mood disorder were higher among women, the single, those living in poverty, the Canadian born and those between 30 and 69 years of age. The presence of comorbid mood disorders was significantly associated with short-term disability, requiring help with instrumental daily activities and suicidal ideation. Health care providers are urged to proactively screen chronically ill patients for mood disorders, particularly among the subgroups found to have elevated risk for these disorders.
\end{abstract}

Key words: Ontario, mood disorders, chronic diseases, quality of life, short-term disability

\section{Introduction}

Mood disorders (major depressive disorder, bipolar disorder, mania or dysthymia) are the most prevalent of all mental disorders. One in 7 adults $(13.4 \%)$ living in Canada reported symptoms that met the criteria for a mood disorder at some point in their lives and about $5.3 \%$ of the Canadian population aged 15 years and over were identified as suffering from a mood disorder in 2002. ${ }^{1}$ The burden of depression to individuals and societies is such that the World Health Organization has projected that by the year 2020, unipolar major depression will be the leading cause of disability-adjusted life years (DALYs) after cardiovascular disease. ${ }^{2}$ Evidence exists that the prevalence of mood disorders in individuals with chronic physical illness is noticeably higher compared to individuals with no such illness. According to the 2002 Mental Health and Well-being Survey, an individual with a chronic physical condition was twice as likely as an individual without such a condition to have a mood disorder. $^{3}$ Depressive disorders often accompany chronic illnesses such as heart disease, stroke, Parkinson's disease, cancer and HIV/AIDS (Evans et al, 2005). ${ }^{4}$ For example, reported prevalence rates of depression range from $17 \%$ to $27 \%$ in patients with cardiac disease, from $22 \%$ to $29 \%$ in patients with cancer and from $9 \%$ to $26 \%$ in patients with diabetes. ${ }^{4}$ Fuller-Thomson and Sulman $^{5}$ reported that among Canadians who had inflammatory bowel disease in 2002, $16.3 \%$ suffered from depression.

High prevalence of mood disorders among individuals with chronic physical conditions represents a significant burden to individuals and society. At the individual level, they can lead to occupational impairment, disruption in interpersonal and family relationships, poor health and suicide. ${ }^{6}$ Existing research suggests that major depression interacts with physical illness to amplify the disability associated with many physical conditions as well as adversely affect the course of physical illnesses. ${ }^{4}$ Individuals with physical health problems often experience anxiety or depression, which affects their response to the treatment of their physical illness. On the other hand, individuals with mental illness can develop physical symptoms and illnesses, such as weight loss and biological disturbances associated with eating disorders. Most of the research done in this field focuses on the impact of depression on quality of life after adjusting for the severity of physical illness (e.g. Vali and Walkup ${ }^{7}$ and Ades, et al. ${ }^{8}$ ) rather than comparing the impact of comorbid mental disorders in individuals with and without physical illness. These studies usually find that depression adversely affect patients' quality of life after adjusting for severity of physical illness. Ferketich, et al. ${ }^{9}$ analyzed data collected in a National longitudinal study and found that depressed men had a $71 \%$ greater risk of developing heart disease and were 2.34 times more likely than non-depressed men

Author References

1 Faculty of Social Work at the University of Toronto

Correspondence: Tahany Gadalla, Assistant Professor, Faculty of Social Work/University of Toronto, 246 Bloor Street West, Toronto, Ontario, Canada M5S 1A1, Tel: (416) 946-0623, Email: tahany.gadalla@utoronto.ca 
to die from this condition. Simon, et al. ${ }^{10}$ compared SF-36 subscale ratings on a clinical sample of depressed patients with and without chronic physical illness and concluded that depressive disorders and chronic physical illness produced differing patterns of impairment, and that comorbid depressive disorders created a substantial burden of additional functional impairment. Katon ${ }^{11}$ estimated medical costs for patients with major depression to be almost $50 \%$ higher than the costs of chronic physical illness alone. The associations between other mood disorders such as bipolar disorder or dysthymia and physical illness and their effect on the quality of life of the physically ill have not been examined.

At the national level, comorbid mood disorders can adversely affect the economy through reduced productivity and higher health care costs. Several studies have investigated the impact of comorbid depressive disorders on the cost of health services using patients with specific physical conditions such as arthritis. ${ }^{7}$ In a study of the economic burden of mental health in Canada, Stephens and Joubert ${ }^{12}$ estimated work-related productivity losses due to mood disorders alone to be $\$ 4.5$ billion annually. Little is known about the impact of comorbid mood disorders on individuals' productivity, daily functioning and quality of life in the Canadian population. Further, the cost to society of the comorbidity of mental disorders and physical conditions requires further investigation.

The widely reported under-treatment of mood disorders magnifies and reinforces their adverse impact on the lives of the physically ill and society as a whole. Although effective interventions for the treatment of mood disorders are available, most individuals who suffer from them fail to consult health professionals. Strakes, et al. ${ }^{13}$ reported that only $40 \%$ of people with probable depression in Atlantic Canada consulted a general practitioner or a mental health specialist about their condition. Despite a universal coverage of physician and hospital services in Canada, only $56.4 \%$ of Canadian women identified as having at least one major depressive episode (MDE) in 2002 reported accessing health care resources in the 12 months prior to the interview. ${ }^{14}$ Among Canadians with inflammatory bowel disease who were identified as having major depression, only $40 \%$ were using antidepressants, and between one third and one half were not consulting any mental health professionals. ${ }^{5}$ Identification of the sociodemographic characteristics of physically ill individuals at high risk of having mood disorders provides health care providers with essential knowledge for targeting high risk individuals leading to early diagnosis and intervention.

Most studies that explored the comorbidity of mental disorders and physical conditions have used clinical samples, which may lead to biased results. Thus, researchers have highlighted the role of populationbased studies in determining the extent and nature of comorbidity between mental disorders and physical illness and the impact of such comorbidity on the afflicted individuals and the society at large. To date, only a few studies which have examined the comorbidity between mental disorders and physical conditions have been conducted in community based samples. In addition, most of these studies used self-reported diagnosis of physical conditions or self-reported symptoms of mental disorders. The present investigation was based on the most recent data available on a representative sample of individuals living in the province of Ontario, Canada. This investigation had two objectives. First, it examined the relationship between socio-demographic characteristics and mood disorders in Ontarians with chronic physical conditions. Second, it assessed short-term disability, limitations in activities of daily living and suicidal ideation in individuals with comorbid mood disorders compared with those with chronic physical illness only.

\section{Methods}

\section{Sample}

This research was based on a subset of the data collected by Statistics Canada in cycle 3.1 of the Canadian Community Health Survey and are available for public use. ${ }^{15}$
The survey was conducted in 2005 and employed a multistage stratified cluster probability sampling in which dwelling was the final sampling unit. The sample was stratified by province, and urban versus rural regions, within province. The survey sample represented approximately $98 \%$ of the Canadian population aged 12 or older who resided in private dwellings in the ten provinces and the three territories. Persons living on Indian Reserves, residents of institutions, fulltime members of the Canadian Armed Forces and residents of certain remote regions were excluded from the survey. Fifty percent of the respondents were interviewed face-to-face using the computerassisted personal interviewing (CAPI) method and $50 \%$ were interviewed by telephone using the computer-assisted telephone interviewing (CATI) method. Cycle 3.1 of the CCHS included a list of common questions to be used in all provinces and a number of lists of optional questions for provinces to select among them. This was done to allow each province to select questions related to their particular needs and priorities. Consequently, not all variables are available for all provinces. Data collected from Ontario participants included all the variables required for the present analysis. Hence, Ontario data were used in this research.

\section{Measures}

Participants were asked the following question, "Now I'd like to ask about certain chronic health conditions which you may have. We are interested in long-term conditions, which are expected to last or have already lasted 6 months or more and that have been diagnosed by a health professional." The list of chronic physical illnesses included food allergies, other allergies, asthma, fibromyalgia, arthritis or rheumatism, back problems, high blood pressure, migraine headaches, chronic bronchitis, emphysema, pulmonary disease, diabetes, epilepsy, cancer, heart disease, bowel disorder, cataracts, glaucoma, thyroid condition, chronic fatigue syndrome, mood disorders (such as major depressive disorder, bipolar disorder, mania or dysthymia), and anxiety disorders (such 
as phobia, obsessive-compulsive disorder or panic disorder).

Two variables were used to measure shortterm disability: the number of disability days during the two weeks prior to the interview and whether the participants required help with their usual daily activities. Survey participants were asked about the number of days they stayed in bed for all or most of the day due to illness or emotional/mental health during the two weeks prior to the interview. They were also asked a series of questions about whether they needed help with instrumental activities of daily living such as preparing meals, shopping for groceries and other necessities, getting to appointments, doing everyday housework, personal care or moving about inside their home because of a long-term health condition. A long-term health condition was defined as a condition that is expected to last or has lasted 6 months or more. In addition, survey participants who live in Ontario were asked whether they had considered suicide in the 12 months prior to the interview.

Socio-demographic characteristics and health indicators used in this research included gender, age group (12 to 29 years, 30 to 49 years, 50 to 69 years, 70 years or older), marital status (married/common law versus divorced/separated/widower/ never married), immigration status (immigrant versus Canadian born), education level (less than secondary school degree, secondary school graduate, some post secondary education and post secondary graduate) and income level (low 30\%, middle $40 \%$ and upper $30 \%$ of the income distribution in Ontario). The income level variable was derived by Statistics Canada to measure the participant's household income relative to the household incomes of all participants living in the province and having the same household size. ${ }^{15}$ First, an adjusted ratio of the participant's total household income to the low-income cutoff corresponding to their household size and community size was calculated. The adjusted ratios were then divided into deciles, i.e. 10 categories including approximately the same percentage of residents.

\section{Data analyses}

Prevalence of comorbid mood disorders among individuals with chronic physical conditions was calculated. Expected probabilities of random co-occurrence of chronic physical conditions and mood disorders were calculated and compared with the observed probabilities. Chi-square tests were used to assess bivariate relationships between prevalence of comorbid mood disorders and socio-demographic characteristics in individuals with chronic physical illness as well as bivariate relationships between mood disorders and quality of life. Logistic regression analysis was used to identify socio-demographic factors associated with a high risk of having mood disorders in individuals with chronic physical illness. Sampling weights were rescaled and used in all analyses. Rescaling the weights to have an average of one has two advantages. First, it takes into account the unequal probabilities of selection of survey participants and adjusts the sample results to the demographic composition of Ontario population so that the results represent the population of Ontario and not just the sample itself. Second, it keeps the total sample size unchanged to guard against inflating the sample size for hypothesis testing.

\section{Results}

Table 1 shows the prevalence of chronic physical illness and mood disorders by gender. Based on data presented in this table, the prevalence of mood disorders among men with chronic physical illness was $6.5 \%$ compared with $1.9 \%$ among men with no such illness. The prevalence of mood disorders among women with chronic physical illness was $10.5 \%$ compared with $3.8 \%$ among women with no chronic physical illness. Using the sampling weights published by Statistics Canada, it can be estimated that approximately 2557000 men and 3170000 women of Ontario residents were living with chronic physical illness in 2005. Among them, approximately 165000 men and 334000 women had been diagnosed with at least one mood disorder.

As shown in Table 1, 10105 of all men (49.3\%) were diagnosed by a health professional as having a chronic physical illness and $4.1 \%$ of all men were diagnosed as having a mood disorder. If these conditions were independent, the probability that they co-occur by chance alone would be $2.02 \%(49.3 \% \times 4.1 \%)$. However, the observed probability of their co-occurrence was $3.18 \%$ indicating an association bet-

TABLE 1

Prevalence of chronic physical illness and mood disorders by gender, Ontario 2005 (sample size $=41$ 701)

\begin{tabular}{|c|c|c|c|c|}
\hline \multirow{2}{*}{\multicolumn{2}{|c|}{ Mood disorders }} & \multicolumn{2}{|c|}{ Chronic physical condition } & \multirow[b]{2}{*}{ Total } \\
\hline & & $\begin{array}{c}\text { Yes } \\
\text { Number (\%) }\end{array}$ & $\begin{array}{c}\text { No } \\
\text { Number (\%) }\end{array}$ & \\
\hline \multirow[t]{3}{*}{ Men } & Yes & $652(6.5 \%)$ & $198(1.9 \%)$ & $850(4.1 \%)$ \\
\hline & No & $9453(93.5 \%)$ & 10191 (98.1\%) & $19644(95.9 \%)$ \\
\hline & Total & 10105 & 10389 & 20494 \\
\hline \multirow[t]{3}{*}{ Women } & Yes & 1319 (10.5\%) & $334(3.8 \%)$ & $1653(7.8 \%)$ \\
\hline & No & 11209 (89.5\%) & 8345 (96.2\%) & $19554(92.2 \%)$ \\
\hline & Total & 12528 & 8679 & 21207 \\
\hline \multirow[t]{3}{*}{ Total } & Yes & $1971(8.7 \%)$ & $532(2.8 \%)$ & $2503(6.0 \%)$ \\
\hline & No & $20662(91.3 \%)$ & $18536(97.2 \%)$ & 39198 (94.0\%) \\
\hline & Total & 22633 & 19068 & 41701 \\
\hline
\end{tabular}


ween the two conditions. Similarly, the observed probability of chronic physical illness and mood disorders in women was found to be $6.22 \%$ compared with a probability of $4.61 \%$ of the two conditions occurring by chance alone.

The prevalence rates of comorbid mood disorders among individuals with different demographic and health characteristics are presented in Table 2. Higher prevalence of mood disorders were found among women, individuals 30 to 49 years of age, lower income groups, Canadian-born, and individuals who were divorced, separated, widowed or never married. The highest proportions of individuals with mood disorders were found among individuals who were diagnosed with chronic fatigue syndrome, fibromyalgia, bowel disorder, stomach or intestinal ulcers, chronic bronchitis or those suffering from the effects of a stroke.
Bivariate chi-square tests indicated that the prevalence of comorbid mood disorders was significantly associated with the individual's gender, age, marital status, immigration status, and education and income levels. Hence, these variables were used in a logistic regression analysis to predict presence of mood disorders in the physically ill. Logistic regression analysis results shown in Table 3 indicate that the odds of having a mood disorder were higher among women, the single, those living in poverty, the Canadian-born and those between 30 and 69 years of age. Chronically ill woman were $65 \%$ more likely than chronically ill men to have mood disorders. The odds of suffering from mood disorders among chronically ill individuals 30 to 49 years of age were almost three times higher than the odds for individuals 70 years or older. Those who were 50 to 69 years old had more than twice the odds of suffering from mood disorders than individuals 70 years of age or older. The odds of having mood disorders for those in the lower $30 \%$ of income distribution were twice the odds of high income individuals. Respondents who were divorced, separated, widowed or never married were $50 \%$ more likely to have mood disorders than those with partners. Education level was not a significant predictor of mood disorders.

Table 4 includes a comparison of quality of life measures in individuals with chronic physical conditions with and without mood disorders. Data presented in this table show that $41.7 \%$ of men with mood disorders had disability days in the two weeks prior to the interview, compared with only $17.7 \%$ without mood disorders ( $p<0.0005)$. Forty-four percent of women with mood disorders reported disability days compared with $22.0 \%$ without mood disorders $(p<0.0005)$. Thirty-six percent

TABLE 2

Prevalence of mood disorders in individuals with chronic conditions by socio-demographic characteristics, Ontario 2005 (sample size $=22$ 633)

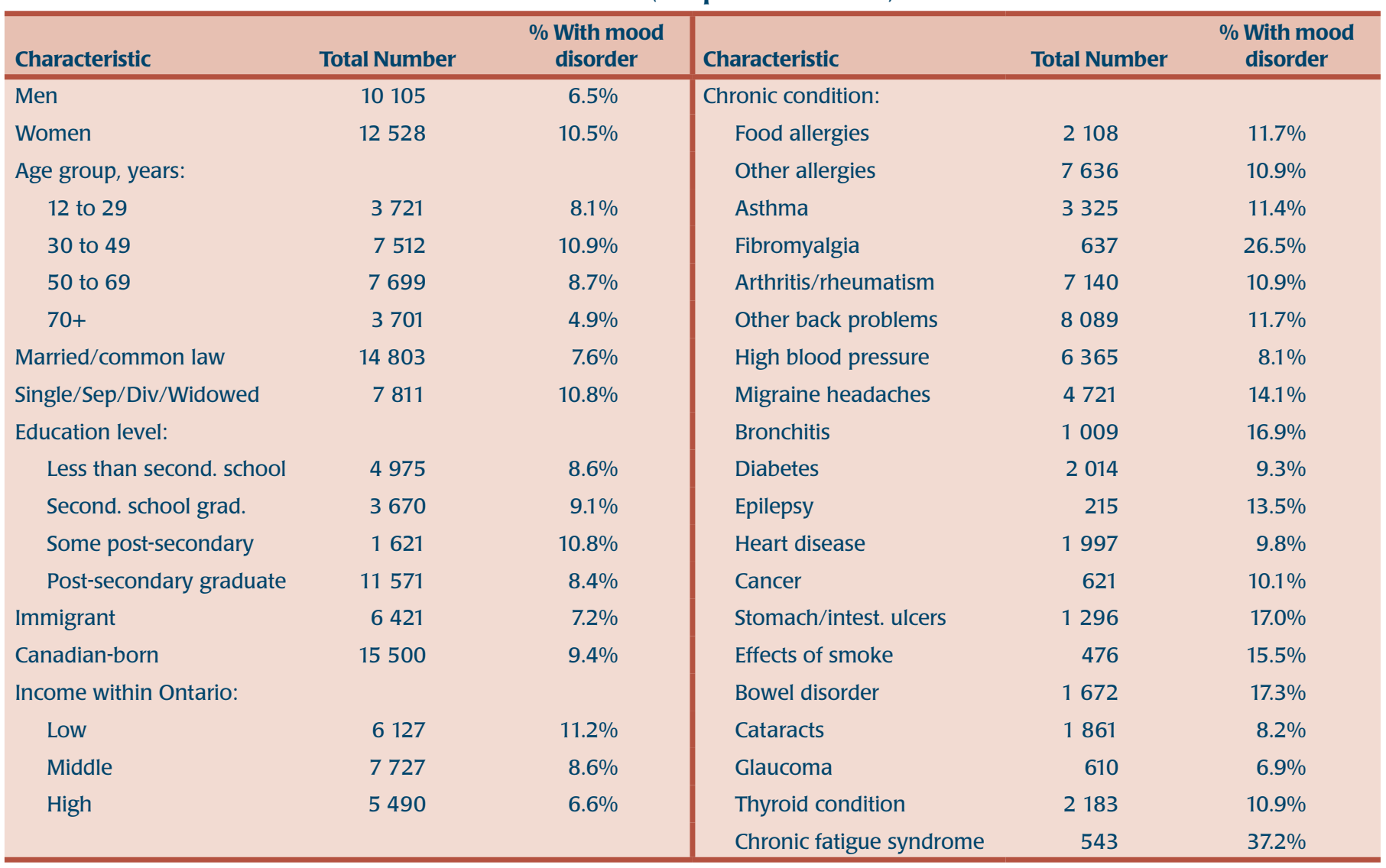


TABLE 3

Socio-demographic factors associated with comorbid mood disorders among individuals with chronic physical illness, Ontario 2005 (sample size $=19213)$

\begin{tabular}{|c|c|c|}
\hline Variable & Odds ratio $(95 \% \mathrm{Cl})$ & $p$-value \\
\hline Female & $1.65(1.49,1.84)$ & $<0.0005$ \\
\hline \multicolumn{3}{|l|}{ Age group, years: } \\
\hline 12 to 29 & $1.42(1.14,1.76)$ & $<0.0005$ \\
\hline 30 to 49 & $2.89(2.40,3.49)$ & $<0.0005$ \\
\hline 50 to 69 & $2.31(1.90,2.79)$ & $<0.0005$ \\
\hline 70+ (reference) & - & \\
\hline Single/Sep/Div/Widowed & $1.51(1.35,1.70)$ & $<0.0005$ \\
\hline Married/common law (reference) & - & \\
\hline Immigrant & $0.64(0.57,0.73)$ & $<0.0005$ \\
\hline Canadian-born (reference) & - & \\
\hline \multicolumn{3}{|l|}{ Income within Ontario: } \\
\hline Low & $1.96(1.70,2.25)$ & 0.002 \\
\hline Middle & $1.38(1.20,1.58)$ & $<0.0005$ \\
\hline High (reference) & - & \\
\hline
\end{tabular}

of chronically ill men and $43.7 \%$ of chronically ill women with mood disorders reported needing help in activities of their daily living such as preparing meals, getting to appointments, doing housework, personal care or moving about inside their home. In contrast, only $15.2 \%$ of men and $25.1 \%$ of women with no comorbid mood disorders reported experiencing such limitations. Data in Table 4 also show that $14.9 \%$ of chronically ill men with mood disorders reported having suicidal thoughts in the 12 months prior to the interview compared with only $1 \%$ of those without a mood disorder $(p<0.0005)$. In women, the percentages were $11.7 \%$ in those with a mood disorder compared to $0.7 \%$ without a mood disorder $(p<0.0005)$. Using the sampling weights published by statistics Canada, it can be estimated that among Ontarians with comorbid mood disorders and chronic physical illness in 2005, approximately 24000 men and 39000 women had suicidal thoughts.

\section{Discussion}

The rates of mood disorders in chronically ill individuals living in Ontario in 2005 ranged between $7.9 \%$ in those with cataracts and $37.2 \%$ in those with chronic fatigue syndrome compared with $2.8 \%$ in individuals with no chronic physical conditions. The prevalence of mood disorders in chronically ill men was more than threefold than in men with no chronic illness. In women with one or more chronic physical condition, it was almost threefold than in non chronically ill women. Prevalence of mood disorders were highest among individuals who had been diagnosed as having fibromyalgia, bowel disorder, stomach or intestinal ulcers, chronic bronchitis or those suffering from the effects of a stroke. Rates of mood disorders in individuals with heart disease, cancer and diabetes observed in this study were lower than those reported in the literature. ${ }^{4}$ This is not surprising since this is a community sample that included those individuals with cancer in remission. Individuals with comorbid mood disorders were more likely to be female, middle aged, living in poverty, without a partner and born in Canada. These characteristics are similar to those of individuals with depression in the general population. ${ }^{16}$

Findings of this investigation showed a highly significant impact of the presence of

\section{TABLE 4}

Relationship between comorbid mood disorders and quality of life of individuals with chronic physical conditions by gender, Ontario 2005 (sample size $=22633$ )

\begin{tabular}{|c|c|c|c|c|}
\hline \multicolumn{5}{|c|}{ Mood disorder } \\
\hline & $\begin{array}{c}\text { Yes } \\
\text { number (\%) }\end{array}$ & $\begin{array}{c}\text { No } \\
\text { number (\%) }\end{array}$ & Chi-square $(\mathrm{df}=1)$ & $p$-value \\
\hline \multicolumn{5}{|l|}{ Men } \\
\hline Disability days in last 2 weeks $\geq 1$ day & $272(41.7 \%)$ & $1671(17.7 \%)$ & 232.4 & $<0.0005$ \\
\hline Need help with daily activities & $234(35.9 \%)$ & $1438(15.2 \%)$ & 189.1 & $<0.0005$ \\
\hline Total - Men & 652 & 9453 & & \\
\hline \multicolumn{5}{|l|}{ Women } \\
\hline Disability days in last 2 weeks $\geq 1$ day & $575(43.6 \%)$ & $2469(22.0 \%)$ & 304.0 & $<0.0005$ \\
\hline Need help with daily activities & $576(43.7 \%)$ & $2814(25.1 \%)$ & 205.2 & $<0.0005$ \\
\hline
\end{tabular}


mood disorders on all measures of quality of life for the chronically ill. The presence of mood disorders was associated with more than double the risk of short-term disability for men and almost double the risk for women. It was also associated with double the proportions of chronically ill men and women requiring help with their daily living activities. In addition, the presence of comorbid mood disorders was associated with an increase in the proportion of those having suicidal ideation 15-fold in men and 17-fold in women.

A bidirectional relationship between mood disorders and chronic physical illness has been proposed that may explain their high comorbidity. ${ }^{4}$ A diagnosis of a disabling physical illness and the associated decline in physical health may cause enough distress to trigger a depressive episode in vulnerable persons. On the other hand, research is discovering that depression itself may act as a risk factor for a variety of chronic illnesses. Goodman and Whitaker (2002) ${ }^{17}$ noted that patients with major depression had higher rates of unhealthy behaviors such as smoking and overeating, which may lead to higher incidence of diabetes and heart disease. Depression has also been shown to be an independent risk factor for type 2 diabetes mellitus. ${ }^{18}$ Perretta, et al. ${ }^{19}$ suggested that depression and mania may act as risk factors for HIV infection by promoting high-risk behaviors. Large population studies indicate that depressed mood or stressful life events may increase the risk of cancer. ${ }^{4}$ In addition, the presence of depression can hinder compliance to treatment and weaken cognitive function, thus, adversely affecting patients' prognosis and increasing their morbidity.

Ample research has focused on trying to understand the mechanisms of the relationship between depression and cardiovascular disease. In addition to promoting unhealthy behaviors and noncompliance with cardiac rehabilitation and medical regimens, some of the possible biologic mechanisms that may explain the increased risk associated with depression in heart disease patients are that depressed patients have decreased heart rate variability, ${ }^{20}$ increased platelet aggregation $^{21}$ and higher levels of inflammatory risk markers. ${ }^{22}$ Other mood disorders may also act as risk factors for physical illnesses and/or adversely affect their prognosis; however, the underlying mechanisms are not as extensively researched as those of depression.

This study has a number of limitations. The identification of individuals as having mood disorders and/or chronic physical illness was not done by clinicians. The survey did not include individuals living in nursing homes, mental institutions or chronic care hospitals, thus, the data underestimate the prevalence of both mood disorders and chronic illnesses. Participants were not asked about each mood disorder separately. Instead, major depressive disorder, bipolar disorder, mania or dysthymia were combined in one question. Consequently, the prevalence and impact of each of these disorders on quality of life cannot be inferred. Further, the cross-sectional nature of the data precluded an examination of the temporal sequence of onset of mood disorders and chronic physical conditions. Given these limitations, the present study determined the prevalence and correlates of mood disorders among individuals with physical chronic illness using the most up-to-date data available on a representative sample of Canadians living in Ontario. In addition, survey respondents were asked to only report mood disorders and physical conditions that had been diagnosed by a health professional. To the authors' knowledge, this is the first study to examine the associations between comorbid mood disorders and short-term disability, limitations of daily activities and suicidal ideation in this population.

Results of this study indicate that detecting and treating depression is as important as treating the physical illness for maintaining quality of life and helping the individual cope with and manage the physical illness. The identification of subgroups of physically ill individuals who are at high risk of suffering from mood disorders provide clinicians with important knowledge for targeting these vulnerable groups for the purpose of early diagnosis and intervention. Health care providers are urged to proactively screen chronically ill patients for mood disorders, particularly among the subgroups found to have elevated risk for these disorders. Based on a survey done by the Depression and Bipolar Support Alliance, ${ }^{23}$ it was concluded that most primary care physicians lacked knowledge about mood disorders in general and bipolar disorder in particular. Findings of this study stressed the importance of providing physicians with more information about the connection between mental and physical health and of including the treatment of the patient's mood disorders as an integral part of the treatment plan of the chronic condition.

It is also imperative to encourage individuals with physical illness to communicate their psychological symptoms to their health care providers and not to accept such symptoms as natural consequence to their physical condition. Barriers to seeking help for mental health, whether perceived or real, should be recognized and removed. The collection of data on cultural and social factors, such as beliefs about stigma and shame in association with mental illness and trust in health care providers, is essential for developing innovative and creative strategies for promoting mental health. Culturally appropriate psychoeducational programs could help Canadians learn to recognize the symptoms of mood disorders and be aware of the importance and potential benefits of treatment. Such programs could promote self-identification and lead to early diagnosis and intervention.

\section{Acknowledgements}

This research was based on data collected and made available by Statistics Canada. The views and opinions expressed do not represent the views of Statistics Canada. 


\section{References}

1. Statistics Canada. Canadian Community Health Survey, Mental Health and Well Being.Public-UseMicrodataDocumentation. Ottawa: Statistics Canada, 2002.

2. Michaud CM, Murray CJ, Bloom BR. Burden of disease: Implications for future research. JAMA. 2001;285:535-539.

3. Public Health Agency of Canada. The Human Face of Mental Health and Mental Illness in Canada. Ottawa: Public Health Agency of Canada, 2006. Cat. No. HP519/2006E.

4. Evans DL, Charney DS, Lewis L, Golden RN, Gorman JM, Krishnan KR, Nemeroff CB, Bremner JD, Carney RM, et al. Mood disorders in the medically ill: Scientific review and recommendations. Biol Psychiatry. 2005;58:175-189.

5. Fuller-Thomson E, Sulman J. Depression and inflammatory bowel disease: findings from two nationally representative Canadian surveys. Inflamm Bowel Dis. 2006;12(8):696-706.

6. Olfson M, Klerman GL. Depressive symptoms and mental health service utilization in a community sample. Soc Psychiatry Psychiatr Epidemiol. 1992;27(4):161-167.

7. Vali F, Walkup J. Combined medical and psychological symptoms: Impact on disability and health care utilization of patients with arthritis. Med Care. 1998; 36:1073-1084.
8. Ades P, Savage P, Tischler M, Poehlman E, Dee J, Niggel J. Determinants of disability in older coronary patients. Am Heart J. 2002;143:151-156

9. Ferketich A, Schwartzbaum J, Frid D, Moeschberger M. Depression as an antecedent to heart disease among women and men in the WHANES I study. National Health and Nutrition Survey. Arch Intern Med. 2000;160:1261-1268.

10. Simon G, Von Kroff M, Rutter C, Paterson D. Treatment process and outcomes for managed care patients receiving new antidepressant prescriptions from psychiatrists and primary care physicians. Arch Gen Psychiatry. 2001;58:395-401.

11. Katon WJ. Clinical and health services relationships between major depression, depressive symptoms and general medical illness. Biol Psychiatry. 2003;54:216-226.

12. Stephens $\mathrm{T}$ \& Joubert N. The economic burden of mental health problems in Canada. Chronic Dis Can. 2001;22(1):18-23.

13. Starkes J, Poulin C, Kisely S. Unmet need for the treatment of depression in Atlantic Canada. Can J Psychiatry. 2005;50:580-90.

14. Gadalla TM. Comparison of users and nonusers of mental health services among depressed women: A National Study. Women\&Health. 2008;47(1):1-19.

15. Statistics Canada. Canadian Community Health Survey, Cycle 3.1, 2005. Public-Use Microdata Documentation. Ottawa: Statistics Canada, 2006.
16. Patten SB. Incidence of major depression in Canada. Can Med Assoc J. 2000; 163:714-715.

17. Goodman E, Whitaker R. A prospective study of the role of depression in the development and persistence of adult obesity. Pediatrics. 2002;110:497-504.

18. Kawakami N, Takatsuka N, Shimizu H, Ishibashi $\mathrm{H}$. Depressive symptoms and occurrence of type 2 diabetes among Japanese men. Diabetes. 1999;22:1071-1076.

19. Perretta P, Akiskal HS, Nisita C, Lorenzetta C, Zaccagnini E, Della Santa M, Cassano GB. The high prevalence of bipolar II and associated cyclothymic and hyperthymic temperaments in HIV-patients. J Affect Disord. 1998;50:215-224.

20. Gormen JM, Sloan RP. Heart rate variability in depressive and anxiety disorders. Am Heart J. 2000;140(4 suppl):77-83.

21. Whyte E, Pollock B, Wagner W, Mulsant B, Ferrel R, Mazumdor S, Reynolds C $3^{\text {rd }}$. Influence of serotonin-transporter-linked promoter region polymorphism on the platelet activation in geriatric depression. Am J Psychiatry. 2001;158:2074-2076.

22. Miller GE, Stetler CA, Carney RM, Freedland KE, Banks WA. Clinical depression and inflammatory risk markers for coronary artery disease. Am J Cardiol. 2002;90:1279-1283.

23. Depression and Bipolar Support Alliance (2002). General Public Survey (Report) Chicago, IL: DBSA 